\title{
Leveraging IgG N-glycosylation quantitative-trait loci to characterize the relationship between Type 2 Diabetes and hypertension: A network with bidirectional mendelian randomization study
}

Di Liu

Capital Medical University

Jie Zhang

Capital Medical University

Xiaoyu Zhang

Capital Medical University

Qiuyue Tian

Capital Medical University

Xiaoni Meng

Capital Medical University

Lijuan Wu

Capital Medical University

Manshu Song

Edith Cowan University

Haifeng Hou

shandong first medical university

Wei Wang

Edith Cowan University

Qun Meng ( $\nabla$ mengqun@nhfpc.gov.cn )

Capital Medical University

Youxin Wang ( $\nabla$ wangy@ccmu.edu.cn )

Capital Medical University

Original investigation

Keywords: Bidirectional Mendelian randomization, Glycosylation, IgG N-Glycosylation quantitative trait loci, Type 2 diabetes, Hypertension

Posted Date: July 8th, 2020 
DOI: https://doi.org/10.21203/rs.3.rs-38821/v1

License: (c) (1) This work is licensed under a Creative Commons Attribution 4.0 International License. Read Full License 


\section{Abstract}

Background: The relationship between IgG N-glycosylation, type 2 diabetes (T2D) and hypertension is not well understood.

Methods: A genome-wide association study (GWAS) of IgG N-glycosylation traits from 536 individuals was performed and 1203 IgG N-glycan quantitative trait loci (IgG N-glycan-QTL) variants targeting 24 IgG $\mathrm{N}$-glycosylation were mapped traits after multi-testing correction. Network with bidirectional mendelian randomization (MR) analysis was performed to examine the causal association between IgG Nglycosylation, T2D and hypertension.

Results: By linking IgG N-glycan-QTL variants with GWAS results for T2D and hypertension, 19 putatively causal IgG N-glycans for T2D and 21 putatively causal IgG N-glycans for hypertension were identified. IgG N-glycan-QTL determined IgG N-glycosylation to higher T2D was associated with higher hypertension risk $(\beta[95 \% \mathrm{Cl}]=1.234$ [0.939-1.529], $\mathrm{P}<0.001)$. In addition, IgG N-glycan-QTL determined IgG Nglycosylation to higher hypertension was associated with higher T2D risk $(\beta[95 \% \mathrm{Cl}]=0.753[0.140-$ 1.3669], $P=0.016)$. No evidence of pleiotropic bias was detected in MR-Egger analysis.

Conclusions: Overall, our study showed that IgG N-Glycosylation-QTLs determined T2D is associated with higher hypertension risk, and vice versa, performing bidirectional regulation through IgG N-Glycosylation.

\section{Background}

Type 2 diabetes (T2D) and hypertension, which are the 2 leading components of the global burden of disease, commonly coexist[1-4]. The coexistence of T2D and hypertension confers a dramatically increased the risk of cardiovascular disease, stroke, and even death, compared with the normotensive and nondiabetic adults $[5,6]$. Increasing evidence suggests epidemiological links between T2D and hypertension $[4,5,7,8]$. Therefore, understanding the pathological and bidirectional links between T2D and hypertension is of significant public health importance about disease prevention and management of complications.

Glycosylation, which is an important and common post-transcriptional modification, is involved in many biologic processes and disease susceptibility $[9,10]$. IgG is the main component of antibody and plays an important role in non-specific immune response. Alternative $\mathrm{N}$-glycosylation markedly affects IgG structure and function and by extension, immune responses, thus acting as a switch between pro- and anti-inflammatory IgG functionality[11, 12]. IgG N-glycosylation has been shown to play an important role in the development of hypertension and T2D[13-15]. However, the relationship between IgG Nglycosylation, T2D and hypertension is not well understood.

Mendelian randomization (MR) analysis, a form of instrumental variable (IV) analysis that leverages the random assortment of genetic variants during gamete formation and, therefore, minimizes the influence of confounding and reverse causation, has been popularly used in estimating causal inference between 
exposures and outcomes[16-18]. However, the current MR often faces the bias of weak IVs due to genetic variation can only explain a small fraction of the exposure variance[19]. Recent studies have incorporated data on genetic variants associated with gene expression (expression quantitative trait loci [eQTL]) into results from GWASs of complex traits to help increase the power of GWAS in identifying loci associated with complex traits[20, 21]. In addition, the method incorporating QTL information into GWAS analyses improves the explanation of complex traits variance by quantitative trait, indicating that QTL variants provides excellent IVs for exposure in MR analysis. This direction of inquiry can be extended to other "omic" data types to gain further insights into the mechanistic pathway between genetic variant and causally associated trait[22, 23]. A recent study showed that variants involved in the regulation of glycoenzymes play an important role in IgG N-glycosylation[24], thus we hypothesized that identifying IgG N-glycosylation quantitative trait loci (IgG N-glycan-QTLs) variants and linking them to diseaseassociated genetic variants from GWAS might pinpoint molecular mechanisms underlying genetic susceptibility to human diseases that are due, at least in part, to altered IgG N-glycosylation.

In addition, the network mendelian randomization aims to investigate more complex networks of relationships between variables, in particular where some of the effect of an exposure on the outcome may operate through a mediator $[25,26]$. Therefore, we performed an analytical framework that integrates network MR and bidirectional MR to explore whether T2D may act as potential mediators that lie in the pathway from aberrant IgG N-glycosylation to increased risk of hypertension (i.e., SNP $\rightarrow \operatorname{lgG} \mathrm{N}$ glycosylation $\rightarrow \mathrm{T} 2 \mathrm{D} \rightarrow$ hypertension) or the causal IgG N-glycosylation driving hypertension in turn leads to T2D (i.e., SNP $\rightarrow$ IgG N-glycosylation $\rightarrow$ hypertension $\rightarrow$ T2D).

\section{Methods}

\section{Study participants and study design}

In the present study, data were collected from a community-based cohort, cross-sectional study from Xuanwu Hospital, in Beijing (from September 2009 to September 2012 of the baseline population). Detailed study design and assessment methods were described in the previous study $[13,27]$. Written informed consent was obtained from each subject at the beginning of the study, and the study has been approved by the Ethics Committee of the Capital Medical University, Beijing, China. The ethics approval was given in compliance with the Declaration of Helsinki.

The network MR study included two major components. First, we explored the casual IgG N-glycosylation for T2D and hypertension and the IgG N-glycosylation-QTLs determined IgG N-glycosylation as instrument variables (IVs) for T2D and hypertension. In our present study, the network MR exploring the causal pathway from IgG N-glycosylation to the outcome was proposed to use the IgG N-glycosylationQTLs determined IgG N-glycosylation as IVs for the mediator (T2D or hypertension). Second, the bidirectional association between T2D and hypertension were taken forward for further analyses to better 
understand the relationship between IgG N-glycosylation, T2D and hypertension. As shown in Figure 1, the framework of the network with bidirectional MR analysis consists of 3 different MR tests that are all described below (I-III). First, the causal effects of IgG

$\mathrm{N}$-glycosylation-QTLs determined IgG N-glycosylation on T2D and hypertension are analyzed (I). Next, the causal effects of IgG N-glycosylation-QTLs determined T2D on hypertension is estimated (II). Finally, the causal effects of IgG N-glycosylation-QTLs determined hypertension on T2D is analyzed (III).

\section{Data collection}

All participants were required to undergo physical examination that included anthropometric and biochemical measurements, as delineated in previous study. After an overnight fasting, two tubes of blood ( $5 \mathrm{~mL}$ ) were collected in the morning by venipuncture. One sample was taken in vacuum negative pressure tubes not containing ethylene diamine tetraacetic acid (EDTA) to acquire serum $(2 \mathrm{~mL})$, which was used to detect the blood biochemistry indexes, and the other sample was taken in vacuum negative pressure tubes containing EDTA. The whole blood was centrifuged at $3000 \mathrm{rpm}$ for $10 \mathrm{~min}$, then the plasma ( $3 \mathrm{~mL}$ ) was separated which was used to measure IgG N-glycosylation and the blood cells $(2 \mathrm{~mL})$ was separated which was used to detect genetic variants. All collected blood samples were processed within $8 \mathrm{~h}$ and stored at $-80^{\circ} \mathrm{C}$ until further measurement.

Demographic characteristics of participants, including age, gender, and ethnicity, were collected by a questionnaire. Anthropometric measurements (height, weight) were conducted with the participants wearing only light indoor clothing and without shoes. Body mass index (BMI) was calculated by the formula: weight $(\mathrm{kg}) /$ height $^{2}\left(\mathrm{~m}^{2}\right)$. Systolic blood pressure (SBP) and diastolic blood pressure (DBP) were measured three times on the right arm in a day with a standard mercury sphygmomanometer and subjects were required to rest at least 5 minutes for each measurement. The participants were then classified into the hypertension group (mean SBP $\geq 140 \mathrm{mmHg}$ or mean DBP $\geq 90 \mathrm{mmHg}$ ) or the normal blood pressure group (mean SBP $<120 \mathrm{mmHg}$ and mean DBP $<80 \mathrm{mmHg}$ )[3]. The fasting blood glucose (FBG) concentrations were measured by the glucose oxidase-peroxidase method (Mind Bioengineering Co. Ltd., Shanghai, China). Diagnosis of T2D was made by physicians according to the $1999 \mathrm{WHO}$ Criteria (FBG greater than or equal to $7.0 \mathrm{mmol} / \mathrm{L}$ )[28].

\section{Genotyping and genotype imputation}

The genotyping procedures were conducted with Illumina Omni Zhonghua chips (Illumina, San Diego, CA, USA). Quality control was conducted as described previously[15]. Genotypes were imputed from the 1000 Genomes Project panel phase 3 based on East Asian population using Michigan Imputation Server. SNP with minor allele frequency (MAF) $>0.01$ and imputation quality ratio $>0.3$ were retained, yielding $7,108,659$ imputed SNPs that were used for further IgG N-glycan-QTL mapping. Based on not facing up with the problem of population stratification, we did not correct the principal component. 


\section{IgG N-glycosylation}

The IgG N-glycan profile analysis was performed by the method of hydrophilic interaction chromatography-Ultra Performance Liquid Chromatography. The protocol of the method was reported as described in detail previously[29]. Finally, 24 glycan peaks (GPs, GP1-GP24) were used for further IgG Nglycan-QTL mapping. The structures of glycans in each peak were reported as described in detail previously[29]. For controlling the experimental variability, we adopted normalization methods and batch correction to process the glycan data so that all samples are comparable.

\section{Statistical and bioinformatics analysis}

The first MR analyses was aimed to evaluate the potential causal relationship IgG N-glycans of T2D and hypertension. IgG N-glycans-QTLs analysis was performed to select IVs for 24 IgG N-glycans. Briefly, linear regression was conducted to test the association between each SNP and IgG N-glycan, with each IgG N-glycan as the dependent variable of interest and the SNP as the independent variable, adjusted for age, sex and BMI. In addition, the GWAS analysis of T2D and hypertension were performed adjusting the effect of same confounders including age, sex and BMI. A relatively conservative Bonferroni correction was used (i.e., $P<0.05 / 1,000,000=5 \times 10^{-8}$ ). Since various IgG N-glycans are highly correlated, and the mechanism regulating IgG N-glycosylation is not specific[30, 31], we have not been ruled out IgG Nglycan-QTLs overlapped between GPs. However, as many significant IgG N-glycan-QTLs are in high linkage disequilibrium, we pruned the IgG N-glycan-QTLs at LD $r^{2}<0.1$. The LD proxies were defined using 1000 genomes East Asian samples[32]. MR analysis was undertaken by inverse-variance weighted (IVW) regression with IgG N-glycan as the exposure, T2D or hypertension as the outcome, and the relevant IgG $\mathrm{N}$-glycan-QTL variants as the IVs. Then these IgG N-glycan-QTLs corresponding to the casual IgG Nglycan for T2D or hypertension were as IVs for T2D or hypertension.

For forward MR, we used a conventional inverse-variance weighted (IVW) MR analysis, in which the IgG Nglycan-QTL SNPS-T2D estimate was regressed on the SNPs-hypertension estimate with the intercept term set to zero, weighted by the inverse-variance of SNPs-hypertension estimate, and vice versa for reverse MR. The heterogeneity between SNPs was estimated by Cochran Q statistic. Random-effects IVW model was used if heterogeneity existed, otherwise fixed-effects IVW model was performed. We also conducted MR-Egger and weighted median methods of MR analyses to test the robustness of the results. In addition, the MR-Egger method was used to assess the robustness of estimates to potential violations of the standard IV assumptions attributing from the directional pleiotropy. To investigate the influence of outlying or pleiotropic genetic variants, we performed a leave-one out analysis, in which 1 SNP was omitted in turn[33].

Data cleaning and statistical analysis was performed using R version 3.3.3 and PLINK 1.9. $P<0.05$ was considered as suggestive of evidence for a potential association. 


\section{Results}

The average age of the 536 participants included was 47.87 years, $31.53 \%$ were males (Table S1). There were $62(11.57 \%)$ and 164 (30.59\%) participants with T2D and hypertension, respectively. Of note, 58.06\% of T2D patients had hypertension, while $21.95 \%$ of hypertensive participants were T2D. Hypertension was 2.15 times as frequent in patients with diabetes compared with those who did not have diabetes. Moreover, T2D was 3.14 times as frequent in patients with hypertension compared with those without hypertension.

IgG N-glycan-QTL analysis was performed to explore association of SNPs and IgG N-glycans. As shown in Figure 2, we identified $199 \mathrm{lgG}$ N-glycan-QTL SNPs at $P<5 \times 10^{-8}$ for GP1, 23 for GP2, 11 for GP3, 4 for GP4, 25 for GP5, 2 for GP6, 104 for GP7, 2 for GP8, 9 for GP9, 75 for GP10, 2 for GP11, 25 for GP12, 72 for GP13, 4 for GP14, 87 for GP15, 31 for GP16, 19 for GP17, 2 for GP19, 75 for GP20, 10 for GP21, 466 for GP22, 3 for GP23, 2 for GP24. There was no SNP at $P<5 \times 10^{-8}$ associated with T2D and hypertension (Figure S1-2). Therefore, these IgG N-glycan-QTL SNPs which were selected as candidate IVs for IgG N-glycans. As many significant IgG N-glycan-QTLs are in high linkage disequilibrium, we pruned the IgG N-glycan-QTLs at LD $r^{2}<0.1$ to select. Finally, we considered GP1 ( $\left.\mathrm{N}_{\mathrm{SNPs}}=58\right), \mathrm{GP} 2\left(\mathrm{~N}_{\mathrm{SNPs}}\right.$ $=8), \mathrm{GP} 3\left(N_{S N P s}=6\right), \mathrm{GP} 4\left(N_{S N P s}=4\right), \mathrm{GP} 5\left(N_{S N P s}=12\right), \mathrm{GP} 6\left(N_{S N P S}=2\right), \mathrm{GP} 7\left(N_{S N P S}=5\right), \mathrm{GP} 8\left(N_{S N P s}=\right.$ $1), G P 9\left(N_{S N P s}=1\right), G P 10\left(N_{S N P s}=4\right), G P 11\left(N_{S N P s}=2\right), G P 12\left(N_{S N P s}=9\right), G P 13\left(N_{S N P s}=5\right), G P 14\left(N_{S N P s}\right.$ $=3), \mathrm{GP} 15\left(\mathrm{~N}_{\mathrm{SNPS}}=2\right), \mathrm{GP16}\left(\mathrm{N}_{\mathrm{SNPs}}=4\right), \mathrm{GP17}\left(\mathrm{N}_{\mathrm{SNPS}}=5\right), \mathrm{GP} 19\left(\mathrm{~N}_{\mathrm{SNPS}}=2\right), \mathrm{GP} 20\left(\mathrm{~N}_{\mathrm{SNPs}}=2\right), \mathrm{GP} 21$ $\left(N_{S N P s}=3\right), G P 22\left(N_{S N P s}=109\right), G P 23\left(N_{S N P s}=1\right)$, and GP24 $\left(N_{S N P s}=1\right)$. The identified IgG N-glycanQTLs as IVs for each IgG N-glycan trait were strongly associated with IgG N-glycosylation, and the mean $( \pm S D)$ proportions of interindividual variation in IgG N-glycan explained by the single SNP (\%) was $5.07 \pm$ 1.04 (Table S2).

As shown in Table S3, MR analysis identified 19 putatively causal IgG N-glycans (GP1-2, GP4-6, GP9-12, GP14-24) for T2D. While 21 lgG N-glycans including GP1, GP3-9, GP11-24 were found to be putatively causal for hypertension (Table S4). GP2 and GP10 were found to be only causal for T2D, and GP3, GP7-8, GP13 were found to be only causal for hypertension. In addition, there was 17 causal IgG N-glycans overlapped between T2D and hypertension.

We further performed a bidirectional MR to evaluate the causal associations between T2D and hypertension. For forward MR analysis, we used the causal IgG N-glycan-QTL SNPs associated with T2D as IVs $\left(\mathrm{N}_{\mathrm{SNPs}}=182\right)$. As shown in Table 1, the IgG N-glycan-QTL determined IgG N-glycosylation to higher T2D was associated with higher hypertension risk $(\beta[95 \% \mathrm{Cl}]=0.756[0.616-0.897], P<0.001)$ by the method of IVW. The findings were robust different methods. However, MR-Egger regression performed the evidence of directional pleiotropy for the association ( $\beta$ [intercept] $=0.860, P<0.001$ ). Then, we removed the causal IgG N-glycan-QTL SNPs overlapped between T2D and hypertension, and the IgG N-glycan-QTL SNPs which were out of the egger regression line. Finally, we considered 6 causal IgG N-glycan-QTL SNPS as IVs for T2D. The results showed that the IgG N-glycan-QTL determined IgG N-glycosylation to higher 
T2D was associated with higher hypertension risk $(\beta[95 \% \mathrm{Cl}]=1.234[0.939-1.529, P<0.001)$ by the method of IVW. The findings were robust different methods. In addition, MR-Egger regression indicated no evidence of directional pleiotropy for the association ( $\beta$ [intercept] $=0.321, P=0.277$ ).

Taking these putative associations forward, we evaluated the potential for reverse causal relationships by performing MR of hypertension against T2D. For reverse MR analysis, we used the casual IgG N-glycanQTLs associated with hypertension $\left(\mathrm{N}_{\mathrm{SNPs}}=186\right)$ as IVs. As shown in Table 2, the IgG N-glycan-QTL determined IgG N-glycosylation to higher hypertension was associated with higher T2D risk $(\beta$ [95\% $\mathrm{Cl}]$ $=0.910[0.772-1.049], P<0.001)$ by the method of IVW. The same finding was found by the method of weighted median MR. However, MR-Egger regression performed the evidence of directional pleiotropy for the association ( $\beta$ [intercept] $=0.850, P<0.001$ ). Then, we removed the causal IgG N-glycan-QTL SNPS overlapped between T2D and hypertension. Finally, we considered 15 causal IgG N-glycan-QTL SNPs as IVs for hypertension. The results showed that the IgG N-glycan-QTL determined IgG N-glycosylation to higher hypertension was associated with higher T2D risk $(\beta[95 \% \mathrm{Cl}]=0.753[0.140-1.366], P=0.016)$ by the method of IVW. The same finding was found by the method of weighted median MR. In addition, MREgger regression performed no evidence of directional pleiotropy for the association ( $\beta$ [intercept] $=0.800$, $P=0.081)$.

\section{Discussion}

To our knowledge, this is the first study to investigate the causal relationship between IgG Nglycosylation, T2D and hypertension using a network with bidirectional MR design integrating IgG NGlycosylation-QTLs and GWAS data. Our study showed that the IgG N-Glycosylation-QTLs determined type 2 diabetes was associated with higher hypertension risk (i.e., SNP $\rightarrow \lg G \mathrm{~N}$ glycosylation $\rightarrow$ T2D $\rightarrow$ hypertension), and vice versa (i.e., SNP $\rightarrow \lg G N$ glycosylation $\rightarrow$ hypertension $\rightarrow$ T2D). We highlighted a causal feedback loop between T2D and hypertension through the regulation of IgG N-Glycosylation.

T2D and hypertension are common comorbidities. The previous bidirectional MR study found T2D may causally affect hypertension, whereas the relationship from hypertension to T2D is unlikely to be causal[34]. However, another MR study showed that genetic increase in SBP increased the risk of T2D [35]. Above all, the evidence for the causal relationship between T2D and hypertension have yielded inconsistent results. In our bidirectional MR study, bi-directional regulation of T2D and hypertension seems biologically plausible. A cohort study found that not only does the presence of hypertension predict future diabetes mellitus, but also the incidence of hypertension increases significantly in the presence of diabetes mellitus through copredication and time trajectories analysis[36]. In addition, a wide range of evidence indicated that T2D and hypertension may be a cause and effect $[4,5,8]$. T2D and hypertension are closely interlinked due to the common risk factors, including obesity, dyslipidemia, endothelial dysfunction, and atherosclerosis[6, 7, 37]. The shared mechanisms of oxidative stress, inflammation, and activation of the immune system also likely contribute to the relationship between T2D and hypertension[7, 38]. 
The IgG N-glycosylation, which plays an important role of the molecular mechanism leading to the promotion of inflammation[11], has been shown to be associated with T2D and hypertension susceptibility[13-15]. In the present MR integrating IgG N-glycosylation-QTL and GWAS data, we found the causal inference of IgG N-glycosylation on T2D and hypertension. The comprehensive IgG N-glycan-QTL resources provided by our study reveal a new richness of detail regarding genetic effects on IgG Nglycosylation patterns and characterize the relationship of IgG N-glycosylation with T2D and hypertension. IgG N-glycosylation provides information that can possibly bridge a GWAS gap regarding disease-related SNPs. The changes of IgG N-glycosylation increased the risk of T2D and hypertension, which could further increase the risk of each other. Our findings were consistent with observational epidemiologic studies, which demonstrated associations of IgG N-glycosylation with T2D and hypertension[13-15], and T2D as a well-known risk factor for hypertension, and vice versa[4, 8, 36, 38].

We didn't filter out SNP associated with T2D and hypertension at $P<5 \times 10^{-8}$. Our method, incorporating IgG N-glycosylation-QTL information into GWAS analyses, has the potential to increase the power of GWAS in identifying loci associated with T2D and hypertension, which was in line with other 'omics'[21, 22]. IgG N-glycans are strongly associated with genetic loci (the proportions of interindividual variation in IgG N-glycan explained by the single SNP in aggregate was 5.07, indicating that QTL variants provides excellent IVs for exposure in MR analysis), might explaining additional phenotypic variation in T2D and hypertension besides genetic variants. Therefore, we explored the casual IgG N-glycosylation for T2D and hypertension with the IgG N-glycosylation-QTL determined IgG N-glycosylation as IVs for T2D and hypertension. In this way, we could explore the causal pathway from IgG N-glycosylation to diseases.

Of note, most of the causal lgG N-glycans for T2D and hypertension were overlapped between T2D and hypertension. Therefore, aside from the bidirectional regulation between T2D and hypertension, we identified that T2D and hypertension share several common genetic and IgG N-glycosylation architectures. The casual IgG N-Glycosylation overlapped between T2D and hypertension might be involved in the bidirectional regulation and underpin these comorbidities. Despite significant advances in our understanding of the pathogenesis and treatment of hypertension, there continues to be debate regarding the pharmacologic treatment of hypertension in patients with diabetes mellitus[38, 39]. Therefore, understanding the pathological links between T2D and hypertension is a critical component of the comprehensive clinical management of disease prevention. Future studies should be focus on the functional network, proposing mechanisms of the regulation of IgG N-Glycosylation on T2D and hypertension.

To the best of our knowledge, the biggest challenge is to integrate multi-omics data to explore the molecular features within the longitudinal landscape not only correlate but causally relate to one another disease[40,41]. The network MR is of the attractive advantage of enabling the interrogation of the potential IgG N-Glycosylation-complex trait to reveal much broader and more complex molecular networks underlying genetic variant-complex disease associations[25]. In addition, our findings provided evidence that endeavors leveraging IgG N-Glycosylation-QTLs data can help to further characterize the complex networks of relationships between IgG N-Glycosylation and complex traits. 


\section{limitations}

This study has several limitations. The cross-sectional nature of our data limits definitive causal inference. The results from MR analyses utilizing genetically predicted that IgG N-glycosylation, T2D and hypertension do not prove causation but provide supportive evidence. Although "multi-omics" data and phenotypic data are measured in the same population to control confounding factors, it limits by the small sample size. In addition, the present MR analyses conducted in participants of Chinese descent might limit the generalization of our findings in other ancestry groups. Finally, statistical power to detect potentially causal relationships through our MR studies was limited for some traits, at least for smaller effects, including some of those observed in our traditional epidemiological analyses.

\section{Conclusion}

In summary, the IgG N-Glycosylation-QTLs determined T2D was associated with higher hypertension risk, and vice versa, performing bidirectional regulation through IgG N-Glycosylation. Evaluation of the genetic and IgG N-Glycosylation overlap between T2D and hypertension can be beneficial to understand the shared biological mechanisms underlying this comorbidity. Future studies are needed to comprehensively characterize the mechanisms of IgG N-Glycosylation, which is involved in T2D and hypertension.

\section{Abbreviations}

95\% Cl: 95\% confidence interval; BMI: body mass index; DBP: diastolic blood pressure; FBG: fasting blood glucose; IVs: instrumental variables; IgG N-glycan-QTL: IgG N-glycan quantitative trait loci; IVW: inversevariance weighted; GPs: glycan peaks; GWAS: genome-wide association studies; LD: linkage equilibrium; MAF: minor allele frequency; MR: Mendelian randomization; QTL: quantitative trait loci; SBP: systolic blood pressure; T2D: Type 2 diabetes.

\section{Declarations}

\section{Acknowledgements}

Not applicable.

\section{Authors' contributions}

$\mathrm{DL}, \mathrm{YW}$, and QM conceived the idea for the study. DL, JZ and XZ obtained the genetic data. DL, XZ, QT and XM performed the data analyses. DL, LW, MS and HH interpreted the results of the data analyses. All authors wrote the manuscript. All authors read and approved the final manuscript.

\section{Competing interests}

The authors declare that they have no competing interests. 


\section{Funding}

The study was supported by grants from the China-Australian Collaborative Grant (NSFC 81561128020 NHMRC APP1112767), and the National Natural Science Foundation of China (81872682 and 81773527). DL was supported by China Scholarship Council (CSC 201908110339).

Availability of data and materials

All data generated or analyzed during this study are included in this published article and its supplementary information files.

Ethics approval and consent to participate Not applicable.

Consent for publication

Not applicable.

\section{References}

1. Ogurtsova K, da Rocha Fernandes JD, Huang Y, Linnenkamp U, Guariguata L, Cho NH, Cavan D, Shaw JE, Makaroff LE: IDF Diabetes Atlas: Global estimates for the prevalence of diabetes for $\mathbf{2 0 1 5}$ and 2040. Diabetes Res Clin Pract 2017, 128:40-50.

2. Mills KT, Bundy JD, Kelly TN, Reed JE, Kearney PM, Reynolds K, Chen J, He J: Global Disparities of Hypertension Prevalence and Control: A Systematic Analysis of Population-Based Studies From 90 Countries. Circulation 2016, 134(6):441-450.

3. Worldwide trends in blood pressure from 1975 to 2015: a pooled analysis of 1479 population-based measurement studies with 19.1 million participants. Lancet 2017, 389(10064):37-55.

4. Emdin CA, Anderson SG, Woodward M, Rahimi K: Usual Blood Pressure and Risk of New-Onset Diabetes: Evidence From 4.1 Million Adults and a Meta-Analysis of Prospective Studies. Journal of the American College of Cardiology 2015, 66(14):1552-1562.

5. de Boer IH, Bangalore S, Benetos A, Davis AM, Michos ED, Muntner P, Rossing P, Zoungas S, Bakris G: Diabetes and Hypertension: A Position Statement by the American Diabetes Association. Diabetes Care 2017, 40(9):1273-1284.

6. Ferrannini E, Cushman WC: Diabetes and hypertension: the bad companions. Lancet 2012, 380(9841):601-610.

7. Petrie JR, Guzik TJ, Touyz RM: Diabetes, Hypertension, and Cardiovascular Disease: Clinical Insights and Vascular Mechanisms. Can J Cardiol 2018, 34(5):575-584.

8. Wei GS, Coady SA, Goff DC, Jr., Brancati FL, Levy D, Selvin E, Vasan RS, Fox CS: Blood pressure and the risk of developing diabetes in african americans and whites: ARIC, CARDIA, and the framingham heart study. Diabetes Care 2011, 34(4):873-879. 
9. Ohtsubo K, Marth JD: Glycosylation in cellular mechanisms of health and disease. Cel/ 2006, 126(5):855-867.

10. Kolarich D, Lepenies B, Seeberger PH: Glycomics, glycoproteomics and the immune system. Curr Opin Chem Biol 2012, 16(1-2):214-220.

11. Shade KTC, Anthony RM: Antibody Glycosylation and Inflammation. Antibodies 2013, 2(3):392-414.

12. Biermann MH, Griffante G, Podolska MJ, Boeltz S, Sturmer J, Munoz LE, Bilyy R, Herrmann M: Sweet but dangerous - the role of immunoglobulin $\mathrm{G}$ glycosylation in autoimmunity and inflammation. Lupus 2016, 25(8):934-942.

13. Wang Y, Klaric L, Yu X, Thaqi K, Dong J, Novokmet M, Wilson J, Polasek O, Liu Y, Kristic J et al: The Association Between Glycosylation of Immunoglobulin $\mathrm{G}$ and Hypertension: A Multiple Ethnic CrossSectional Study. Medicine (Baltimore) 2016, 95(17):e3379.

14. Lemmers RFH, Vilaj M, Urda D, Agakov F, Simurina M, Klaric L, Rudan I, Campbell H, Hayward C, Wilson JF et al: IgG glycan patterns are associated with type 2 diabetes in independent European populations. Biochim Biophys Acta Gen Subj 2017, 1861(9):2240-2249.

15. Ge S, Wang Y, Song M, Li X, Yu X, Wang H, Wang J, Zeng Q, Wang W: Type 2 Diabetes Mellitus: Integrative Analysis of Multiomics Data for Biomarker Discovery. OMICS 2018, 22(7):514-523.

16. Davey SG, Hemani G: Mendelian randomization: genetic anchors for causal inference in epidemiological studies. Hum Mol Genet 2014, 23(R1):R89-98.

17. Verduijn M, Siegerink B, Jager KJ, Zoccali C, Dekker FW: Mendelian randomization: use of genetics to enable causal inference in observational studies. Nephrol Dial Transplant 2010, 25(5):1394-1398.

18. Zheng J, Baird D, Borges MC, Bowden J, Hemani G, Haycock P, Evans DM, Smith GD: Recent Developments in Mendelian Randomization Studies. Curr Epidemiol Rep 2017, 4(4):330-345.

19. VanderWeele TJ, Tchetgen TEJ, Cornelis M, Kraft P: Methodological challenges in mendelian randomization. Epidemiology 2014, 25(3):427-435.

20. Zhu Z, Zhang F, Hu H, Bakshi A, Robinson MR, Powell JE, Montgomery GW, Goddard ME, Wray NR, Visscher PM et al: Integration of summary data from GWAS and eQTL studies predicts complex trait gene targets. Nat Genet 2016, 48(5):481-487.

21. Xu Z, Wu C, Wei P, Pan W: A Powerful Framework for Integrating eQTL and GWAS Summary Data. Genetics 2017, 207(3):genetics.300270.302017.

22. Hannon E, Weedon M, Bray N, O'Donovan M, Mill J: Pleiotropic Effects of Trait-Associated Genetic Variation on DNA Methylation: Utility for Refining GWAS Loci. Am J Hum Genet 2017, 100(6):954959.

23. Ng B, White CC, Klein HU, Sieberts SK, McCabe C, Patrick E, Xu J, Yu L, Gaiteri C, Bennett DA et al: An $\mathrm{XQTL}$ map integrates the genetic architecture of the human brain's transcriptome and epigenome. Nat Neurosci 2017, 20(10):1418-1426.

24. Klaric L, Tsepilov YA, Stanton CM, Mangino M, Sikka TT, Esko T, Pakhomov E, Salo P, Deelen J, McGurnaghan SJ et al: Glycosylation of immunoglobulin $\mathrm{G}$ is regulated by a large network of genes 
pleiotropic with inflammatory diseases. Science advances 2020, 6(8):eaax0301.

25. Burgess S, Daniel RM, Butterworth AS, Thompson SG: Network Mendelian randomization: using genetic variants as instrumental variables to investigate mediation in causal pathways. Int $J$ Epidemiol 2015, 44(2):484-495.

26. Zhan Y, Karlsson IK, Karlsson R, Tillander A, Reynolds CA, Pedersen NL, Hägg S: Exploring the Causal Pathway From Telomere Length to Coronary Heart Disease: A Network Mendelian Randomization Study. Circ Res 2017, 121(3):214-219.

27. Liu D, Chu X, Wang H, Dong J, Ge SQ, Zhao ZY, Peng HL, Sun M, Wu LJ, Song MS et al: The changes of immunoglobulin $\mathbf{G}$ N-glycosylation in blood lipids and dyslipidaemia. J Trans/ Med 2018, 16(1):235.

28. Cruickshank JM: Follow-up of intensive glucose control in type 2 diabetes. N Engl J Med 2009, 360(4):417-418; author reply 418.

29. Liu D, Xu X, Li Y, Zhang J, Zhang X, Li Q, Hou H, Li D, Wang W, Wang Y: Immunoglobulin G N-Glycan Analysis by Ultra-Performance Liquid Chromatography. J Vis Exp 2020(155).

30. Pucic M, Knezevic A, Vidic J, Adamczyk B, Novokmet M, Polasek O, Gornik O, Supraha-Goreta S, Wormald MR, Redzic I et al: High throughput isolation and glycosylation analysis of IgG-variability and heritability of the IgG glycome in three isolated human populations. Mol Cell Proteomics 2011, 10(10):M111 010090.

31. Benedetti E, Pucic-Bakovic M, Keser T, Wahl A, Hassinen A, Yang JY, Liu L, Trbojevic-Akmacic I, Razdorov $\mathrm{G}$, Stambuk $\mathrm{J}$ et al: Network inference from glycoproteomics data reveals new reactions in the IgG glycosylation pathway. Nat Commun 2017, 8(1):1483.

32. Zheng J, Erzurumluoglu AM, Elsworth BL, Kemp JP, Howe L, Haycock PC, Hemani G, Tansey K, Laurin C, Pourcain BS et al: LD Hub: a centralized database and web interface to perform LD score regression that maximizes the potential of summary level GWAS data for SNP heritability and genetic correlation analysis. Bioinformatics 2017, 33(2):272-279.

33. Burgess S, Thompson SG: Interpreting findings from Mendelian randomization using the MR-Egger method. Eur J Epidemio/ 2017, 32(5):377-389.

34. Sun D, Zhou T, Heianza Y, Li X, Fan M, Fonseca V, Qi L: Type 2 Diabetes and Hypertension: A Study on Bidirectional Causality. Circulation Research

35. Aikens RC, Zhao W, Saleheen D, Reilly MP, Epstein SE, Tikkanen E, Salomaa V, Voight BF: Systolic Blood Pressure and Risk of Type 2 Diabetes: A Mendelian Randomization Study. Diabetes 2017, 66(2):543-550.

36. Tsimihodimos V, Gonzalez-Villalpando C, Meigs JB, Ferrannini E: Hypertension and Diabetes Mellitus: Coprediction and Time Trajectories. Hypertension (Dallas, Tex : 1979) 2018, 71(3):422-428.

37. Anfossi G, Russo I, Doronzo G, Trovati M: Contribution of insulin resistance to vascular dysfunction. Archives of physiology and biochemistry 2009, 115(4):199-217. 
38. Cryer MJ, Horani T, DiPette DJ: Diabetes and Hypertension: A Comparative Review of Current Guidelines. Journal of clinical hypertension (Greenwich, Conn) 2016, 18(2):95-100.

39. Yandrapalli S, Pal S, Nabors C, Aronow WS: Drug treatment of hypertension in older patients with diabetes mellitus. Expert opinion on pharmacotherapy 2018, 19(7):633-642.

40. Hawe JS, Theis FJ, Heinig M: Inferring Interaction Networks From Multi-Omics Data. Front Genet 2019, 10:535.

41. Huang S, Chaudhary K, Garmire LX: More Is Better: Recent Progress in Multi-Omics Data Integration Methods. Front Genet 2017, 8:84.

\section{Tables}

Table 1 Causal association between IgG N-glycan-QTLs determined T2D and hypertension

\begin{tabular}{|l|l|l|l|l|l|}
\hline MR_method & Parameter & SNP(N) & Effect (95\%CI) & SE & P \\
\hline IVW & $\beta$ & 182 & $0.756(0.616-0.897)$ & 0.072 & $<0.001$ \\
\hline MR_Egger & $\beta$ & 182 & $0.143(0.039-0.247)$ & 0.053 & 0.007 \\
\hline & $\beta$ (intercept) & 182 & $0.860(0.740-0.980)$ & 0.061 & $<0.001$ \\
\hline Weighted median & $\beta$ & 182 & $0.778(0.670-0.885)$ & 0.055 & $<0.001$ \\
\hline IVW & $\beta$ & 11 & $1.189(0.707-1.672)$ & 0.246 & $<0.001$ \\
\hline MR_Egger & $\beta$ & 11 & $0.227(-0.020-0.475)$ & 0.126 & 0.071 \\
\hline & $\beta$ (intercept) & 11 & $0.948(0.687-1.208)$ & 0.133 & $<0.001$ \\
\hline Weighted median & $\beta$ & 11 & $1.181(0.793-1.569)$ & 0.198 & $<0.001$ \\
\hline IVW & $\beta$ & 6 & $1.234(0.939-1.529)$ & 0.151 & $<0.001$ \\
\hline MR_Egger & $\beta$ & 6 & $0.928(0.385-1.472)$ & 0.277 & 0.001 \\
\hline & $\beta$ (intercept) & 6 & $0.321(-0.258-0.900)$ & 0.295 & 0.277 \\
\hline Weighted median & $\beta$ & 6 & $1.208(0.729-1.687)$ & 0.244 & $<0.001$ \\
\hline
\end{tabular}

Beta is the estimated effect size.

CI: confidence intervals; IVW, inverse-variance weighted; QTL: quantitative trait loci; MR mendelian randomization; SE, standard error; SNP, single-nucleotide polymorphism; T2D, type 2 diabetes.

Table 2 Causal association between IgG N-glycan-QTLs determined hypertension and T2D

\begin{tabular}{|l|l|l|l|l|l|}
\hline MR_method & Parameter & SNP(N) & Effect (95\%CI) & SE & P \\
\hline IVW & $\beta$ & 186 & $0.910(0.772-1.049)$ & 0.071 & $<0.001$ \\
\hline MR_Egger & $\beta$ & 186 & $0.204(-0.115-0.523)$ & .163 & 0.210 \\
\hline & $\beta$ (intercept) & 186 & $0.850(0.497-1.202)$ & 0.180 & $<0.001$ \\
\hline Weighted median & $\beta$ & 186 & $1.002(0.864-1.140)$ & 0.070 & $<0.001$ \\
\hline IVW & $\beta$ & 15 & $0.753(0.140-1.366)$ & 0.313 & 0.016 \\
\hline MR_Egger & $\beta$ & 15 & $-0.018(-1.152-1.115)$ & 0.578 & 0.975 \\
\hline & $\beta$ (intercept) & 15 & $0.800(-0.100-1.699)$ & 0.459 & 0.081 \\
\hline Weighted median & $\beta$ & 15 & $0.961(0.593-1.329)$ & 0.188 & $<0.001$ \\
\hline
\end{tabular}

Beta is the estimated effect size. 


\section{Supplemental Legends}

\section{Figure. S1 Manhattan plot illustrating observed GWAS for T2D}

Points represent $-\log 10 P$ values (y axis) for genetic variants according to their genomic location ( $\mathrm{x}$ axis). Effects that survived the multiple-testing threshold in our analysis $\left(P<5 \times 10^{-8}\right.$ - represented by the red horizontal line) are colored.

GWAS, genome-wide association study; T2D: type 2 diabetes

\section{Figure. S2 Manhattan plot illustrating observed GWAS for hypertension}

Points represent $-\log 10 P$ values (y axis) for genetic variants according to their genomic location (x axis). Effects that survived the multiple-testing threshold in our analysis $\left(P<5 \times 10^{-8}\right.$ - represented by the red horizontal line) are colored.

GWAS, genome-wide association study

\section{Figures}

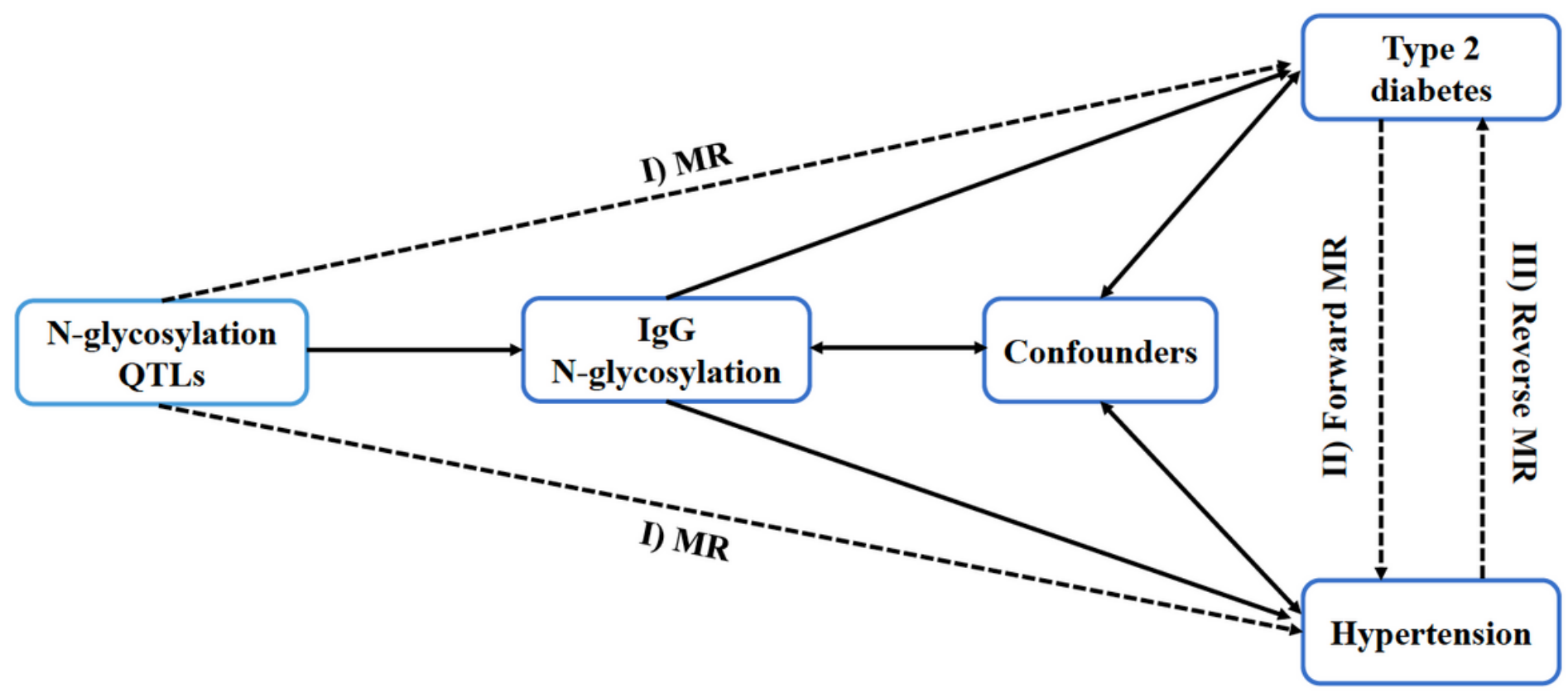


Figure 1

Network with bidirectional Mendelian randomization analysis framework. The solid lines depict the true potential causal diagram. The dashed lines represent the parameters that need to be estimated. MR, mendelian randomization; QTL, quantitative trait loci

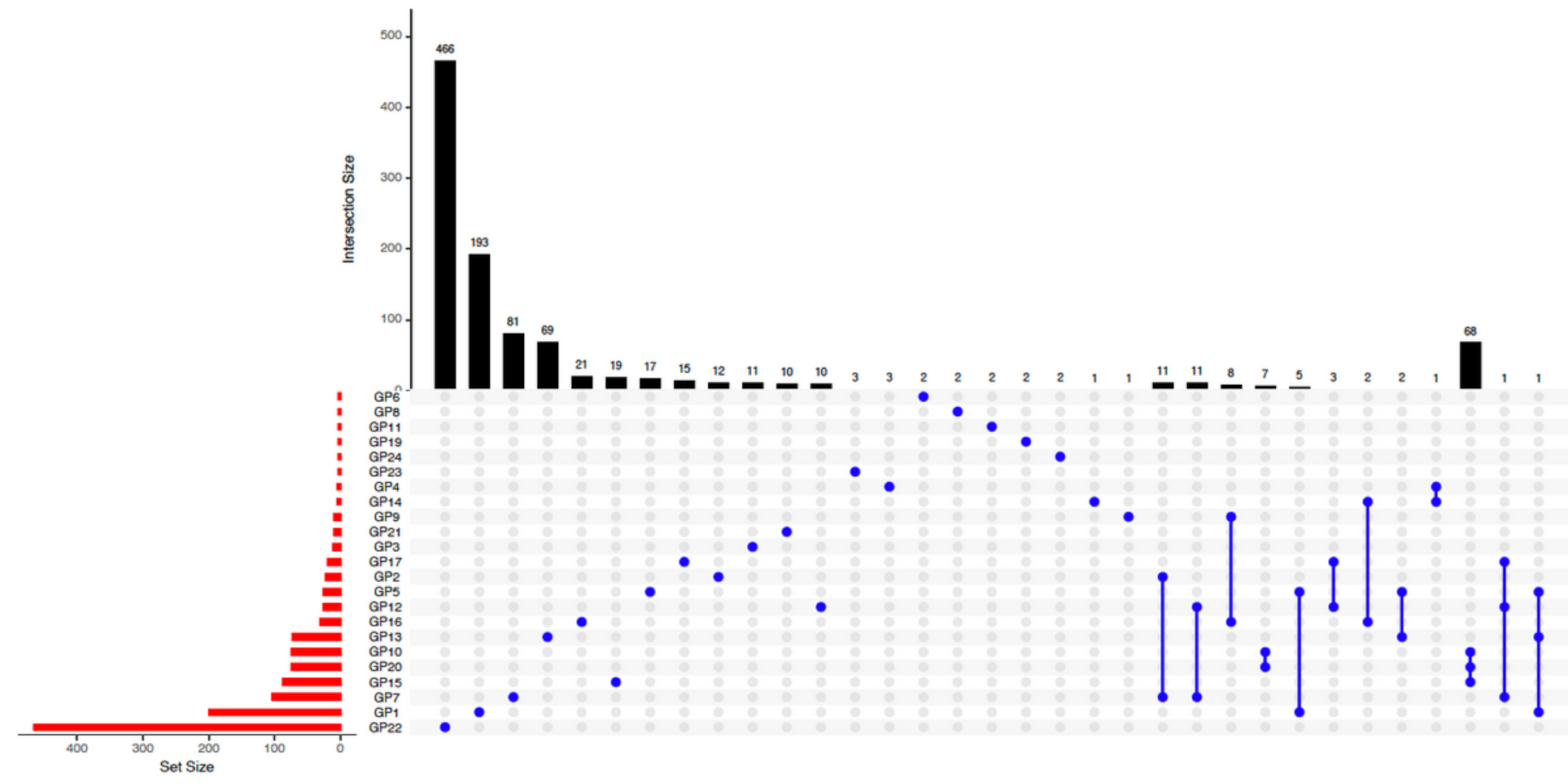

Figure 2

Upset plot for the IgG N-glycan-QTLs overlapping between GPs The plot was performed by R package "UpSetR". The number of IgG N-glycan-QTLs overlapped between GPs is shown. GPs, glycan peaks; QTL, quantitative trait loci 


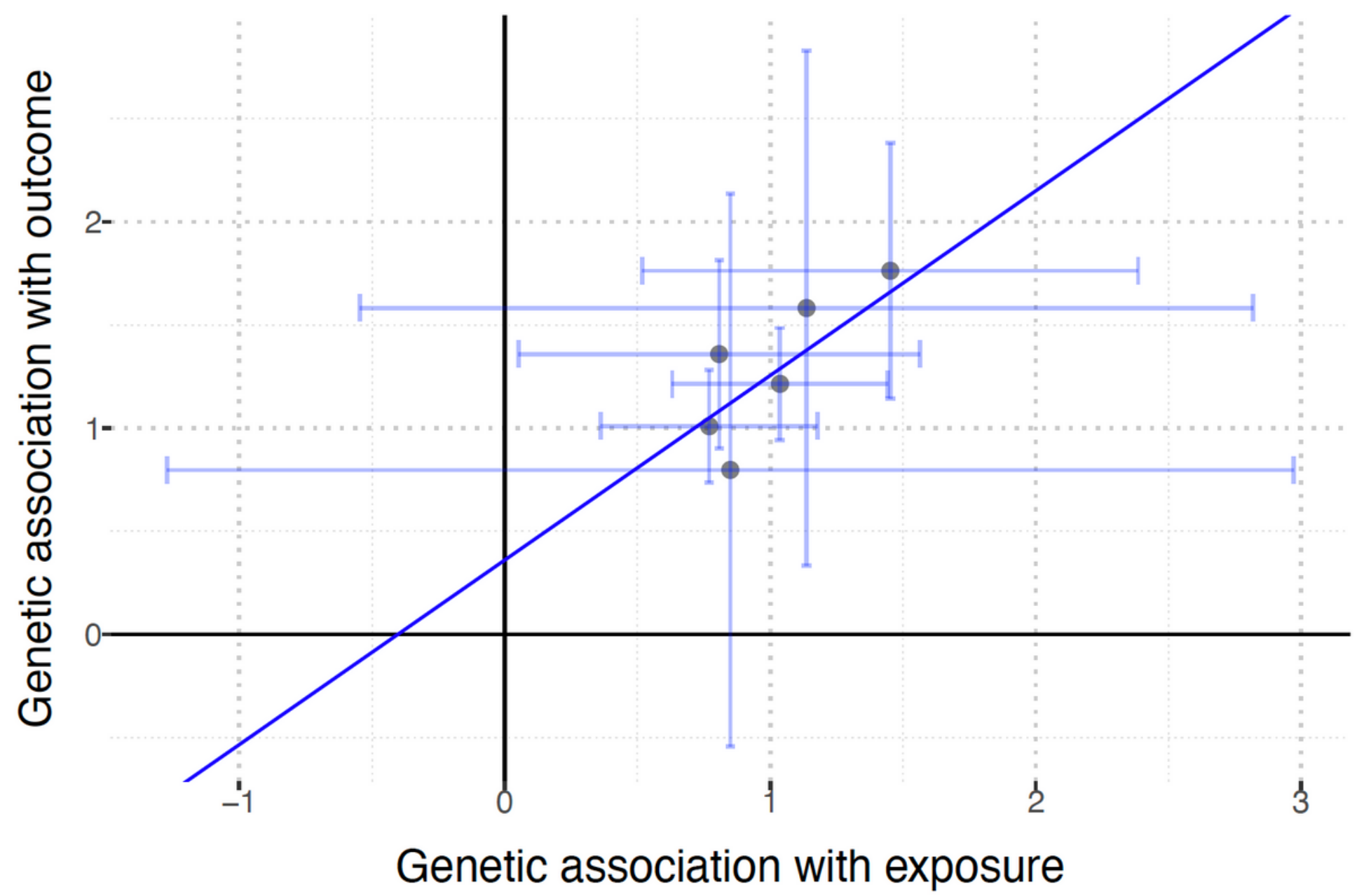

Figure 3

MR plot for associations of T2D variants with risk of hypertension. The red line indicates the estimate of effect using MR-egger method. Circles indicate marginal IgG N-glycan-QTLs associations with T2D and risk of hypertension for each variant. Error bars indicate $95 \%$ Cls. MR, mendelian randomization; QTL, quantitative trait loci; T2D: type 2 diabetes 


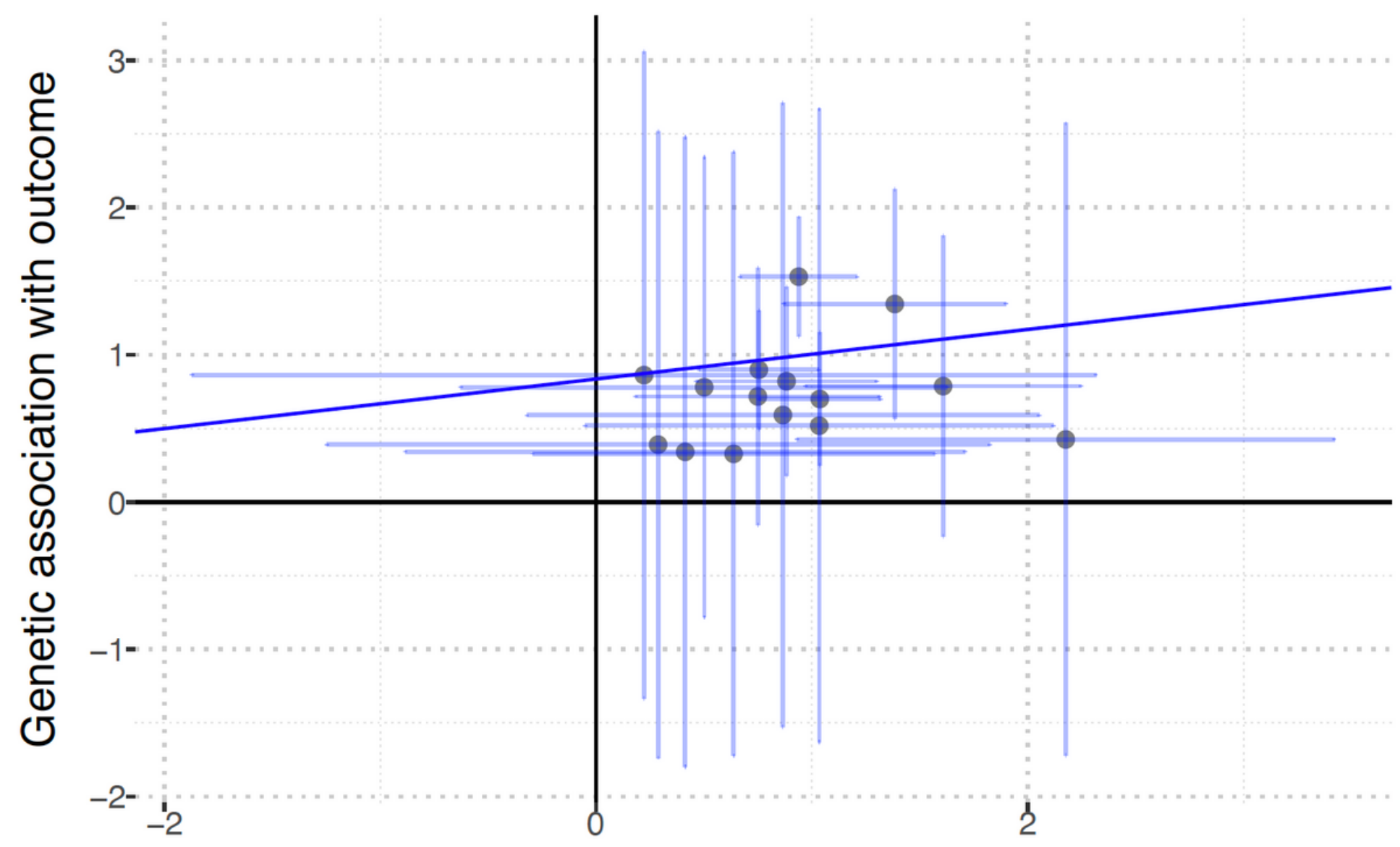

Genetic association with exposure

Figure 4

MR plot for associations of hypertension variants with risk of T2D. The red line indicates the estimate of effect using MR-egger method. Circles indicate marginal IgG N-glycan-QTLs associations with hypertension and risk of T2D for each variant. Error bars indicate $95 \%$ Cls. MR, mendelian randomization; QTL, quantitative trait loci; T2D: type 2 diabetes

\section{Supplementary Files}

This is a list of supplementary files associated with this preprint. Click to download.

- FigureS1.png

- FigureS2.png 